\title{
BRAFV600/PI3K Inhibitor ASN003
}

National Cancer Institute

\section{Source}

National Cancer Institute. BRAFV600/PI3K Inhibitor ASN003. NCI Thesaurus. Code C134967.

A selective inhibitor of mutated forms of B-RAF kinase at amino acid position 600 (BRAFV600), including BRAFV600E, the alpha, delta and, to a lesser extent, beta isoforms of phosphatidylinositide 3-kinase (PI3K), including mutated forms of PI3KCA, which encodes the p110-alpha catalytic subunit of the class I PI3K, and the phosphatase and tensin homologs (PTEN) with loss-of-function mutation, with potential antineoplastic activity. Upon administration of ASN003, this agent selectively targets, binds to and inhibits the activity of BRAFV600 mutants as well as mutated isoforms of PI3K. This inhibits signaling through B-RAF- and PI3K/mechanistic target of rapamycin (mTOR)mediated pathways and inhibits cellular proliferation in tumor cells with BRAFV600 mutations, those expressing PI3K and/or those driven by PTEN. Dysregulation of the BRAF- and PI3K-mediated pathways is frequently seen in a variety of tumors and results in increased tumor cell growth and survival. Dual targeting of both pathways may increase efficacy and anti-tumor potential compared to the targeting of just one pathway by a selective B-RAF inhibitor or selective PI3K pathway inhibitor alone. 